\title{
Acoustoelectric transient spectroscopy of microwave treated GaAs-based structures
}

\author{
O.Ya. Olikh \\ Kyiv Taras Shevchenko National University, Physics Faculty, 6, pr. Glushkova, 03127 Kyiv, Ukraine, \\ Phone: +380 (44) 2660510, E-mail: olikh@mail.univ.kiev.ua
}

\begin{abstract}
The effects of microwave $(2.45 \mathrm{GHz})$ treatment influence on the cross section for electron capture and the energy of the deep levels in the forbidden gap of GaAs monocrystals and $n-n^{+}$epitaxial structures have been investigated using acoustoelectric transient spectroscopy.
\end{abstract}

Keywords: microwave treatment, deep level, gallium arsenide.

Paper received 30.07.03; accepted for publication 11.12.03.

\section{Introduction}

The external factors influence on semiconductors and semiconductor-based structures is widely studied, both to understand degradation processes into semiconductor devices and to find new technological decisions of the such devices fabrication. The influence of some factors, such as radiation, is studied completely enough [1,2]. In recent years, a number of papers are devoted to the new influence methods, such as ultrasonic treatment $[3,4]$ or microwave (MW) electromagnetic irradiation [5-7]. For example, it is shown that MW treatment leads to relaxation of the internal mechanical strain of the Schottkybarrier GaAs structures [5], redistribution of the impurities [5,6], gettering of the point defects [7]. However, information about microwave influence on the structure defect parameters is practically unknown.

In this paper, we present results of investigation MW irradiation influence on the parameters of deep levels located into the under-surface region of $n$-GaAs monocrystals and $\mathrm{GaAs} n-n^{+}$epitaxial structures.

\section{Samples and experimental technique}

The following three types of samples were investigated in this work:

1) The monocrystal GaAs wafer. The (100) wafer, $300 \mu \mathrm{m}$ thick, was doped with $\mathrm{Sn}$. The electron concentration was $(1.5 \div 2.5) \times 10^{18} \mathrm{~cm}^{-3}$ (for the sample labeled as GA1) or $(3 \div 5) \times 10^{16} \mathrm{~cm}^{-3}$ (sample GA2). The (111) wafer, $300 \mu$ m thick, doped with Te, $n=(1 \div 2) \times 10^{18} \mathrm{~cm}^{-3}$, labeled GA3;

2) The GaAs epitaxial $n-n^{+}$structures. The Te-doped GaAs epitaxial layer (free carrier concentration was $3.9 \times 10^{15} \mathrm{~cm}^{-3}$ for the sample GAE1, $3.5 \times 10^{15} \mathrm{~cm}^{-3}$ for GAE2 and $5 \times 10^{15} \mathrm{~cm}^{-3}$ for GAE3) grown on monocrystal Te-doped GaAs substrate $\left(n=2 \times 10^{18} \mathrm{~cm}^{-3}\right)$. The thicknesses of the layer and the substrate were 6 and $300 \mu \mathrm{m}$, respectively.

3) The GaAs:Te epitaxial $n-n^{+}$structures with a buffer layer. The layer, $1 \mu \mathrm{m}$ thick, $n=8 \times 10^{16} \mathrm{~cm}^{-3}$, and the layer, $2 \mu \mathrm{m}$ thick, $n=7 \times 10^{15} \mathrm{~cm}^{-3}$, grown consistently on (100) GaAs substrate $\left(n=2 \times 10^{18} \mathrm{~cm}^{-3}\right)$. The GAB1 and $G A B 2$ samples are made from different wafers.

All investigated epitaxial structures were fabricated using the industrial vapor phase epitaxy method and possessed standard technical characteristics.

Samples were irradiated in free space at the room temperature using magnetron radiation. The microwave frequency was $2.45 \mathrm{GHz}$, the specific power density was $1.5 \mathrm{~W} / \mathrm{cm}^{2}$. The total treatment time was 20 to 60 seconds for different samples; the influence continuous duration was $5-10$ s to avoid sample heating.

The parameters of deep centers (the electron capture cross section $\sigma_{n}$ and the energy depth of the electron trap level counted from the edge of conductivity band $\left.\left(E_{C}-E_{t}\right)\right)$ have been determined before and after the microwave treatment. The method of acoustoelectric tran- 


\section{O.Ya. Olikh: Acoustoelectric transient spectroscopy of microwave ...}

sient spectroscopy is used [8,9]: the GaAs samples are placed by the epitaxial layer on the piezoelectric plate ( $\mathrm{LiNbO}_{3}$ - see Fig.1); during propagation of acoustic waves in the plate, the direct transverse acoustoelectric voltage (TAV) arose in semiconductor samples. After an ultrasonic impulse ending, TAV decreased to zero. This decrease can be interpolated by the exponential function; the characteristic relaxation time $\tau$ is known to be determined by parameters of the deep level, located in the near-surface region $[8,10]$ :

$$
\tau=\left(\sigma_{n} v_{T} N_{c}\right)^{-1} \exp \left(\left(E_{C}-E_{t}\right) / k T\right)
$$

where $v_{T}$ represents the free electron thermal velocity; $N_{c}$ is the effective density of states at the bottom of the conductivity band. The experimental measuring of TAV kinetic at different temperatures allowed to define dependence $\tau(T)$. The angle of the declination of a plot $\ln (\tau)$ on $(k T)^{-1}$ allowed to determine $\left(E_{C}-E_{t}\right)$ and then to calculate $\sigma_{n}$ by using the equation (1).

Our measurements were carried out in the temperature range 290 to $350 \mathrm{~K}$ except for GAB1 and GAB2; for these samples TAV appeared after sample heating up to $310 \mathrm{~K}$ only.

\section{Experimental results and discussion}

Fig. 2 represents the typical $\tau$ temperature dependencies for the two samples before and after MW irradiation. It is visible, that both the plot slopes (related to $\left(E_{C}-E_{t}\right)$ ) and the characteristic relaxation time value changed after the microwave treatment. The other samples dependencies are similar; all results are presented in the Table.

The obtained results have some features. At first, the irradiation influence on the carrier trapping cross section is considerably stronger than the influence on the energy level position. It is confirmed by $\sigma_{n}$ can change value by an order as well as, for example, by $\left(E_{C}-E_{t}\right)$ did not change practically, while $\sigma_{n}$ increased by a factor of 4 approximately at GAB1 after $20 \mathrm{~s}$ MW influence.

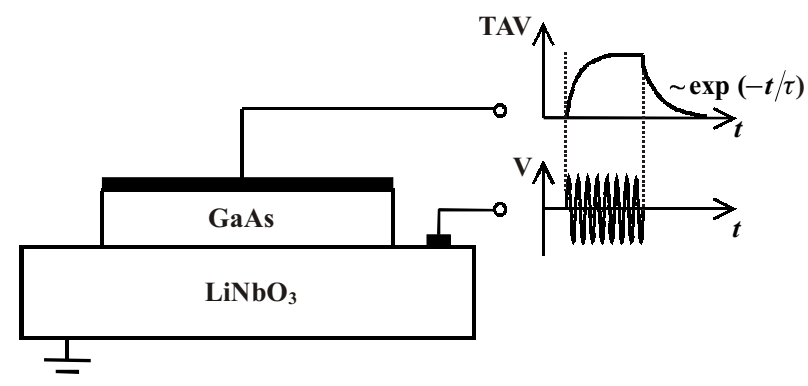

Fig. 1. System for TAV signal measurements. Shown in the insert is the time dependence of the pulse high-frequency $(2.6 \mathrm{MHz})$ voltage $V$ for existing of with ultrasound in the piezoelectric plate and the resulting TAV signal.

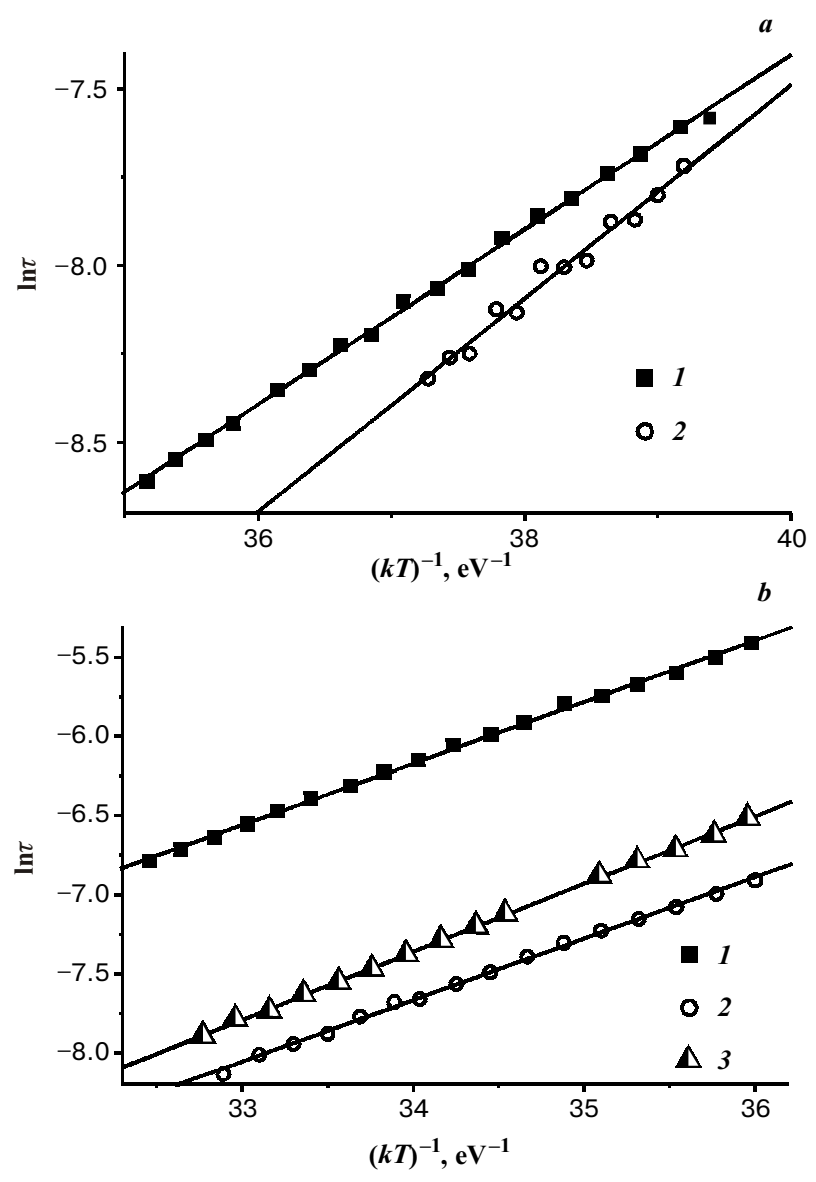

Fig. 2. Temperature dependence of the characteristic TAV relaxation time for the samples $\operatorname{GAE} 2(a)$ and GAB1 $(b)$. The curve 1 corresponds to the unirradiated samples, curves $2-3$ - to the irradiated samples. The microwave treatment time is $20 \mathrm{~s}$ (curve 2,b), $40 \mathrm{~s}$ (curve 3,b), $60 \mathrm{~s}$ (curve 2, a).

Secondly, the irradiation dose for a substantial center parameters changing for the epitaxial structures is higher than for monocrystalline samples: it is possible to compare results for the samples of the GA-set and GAB-set after $20 \mathrm{~s}$ irradiation. After the repeated $20 \mathrm{~s}$ irradiation of the GA sample, the TAV signal diminished considerably and exceed the measuring range. It correlates with data obtained in [7]; this work reports of the decrease in the concentration of the centers with energy level at the forbidden band top half due to microwave annealing. Thirdly, the changes character at the monocrystalline wafers and at the epitaxial structures is different: $\left(E_{C}-E_{t}\right)$ and $\sigma_{n}$ decrease in monocrystals and increase at structures with a buffer layer as well as without it. Finally, the MW stimulated changes degree depend on the free carrier concentration. It is possible to compare results for the GA1 and GA2; these samples differ by the free carrier concentration value only.

It should be noted that although the GaAs levels subjacent a conductivity band bottom on $0.25,0.31,0.33$, 
Table. Parameters of deep levels in GaAs structures before and after the microwave treatment.

\begin{tabular}{|c|c|c|c|}
\hline Sample & $\begin{array}{l}\text { Total time } \\
\text { of microwave } \\
\text { treatment, s }\end{array}$ & $\left(E_{C}-E_{t}\right), \mathrm{eV}$ & $\sigma_{n}{ }^{*}, \mathrm{~cm}^{2}$ \\
\hline \multirow[t]{2}{*}{ GA1 } & 0 & 0.31 & $3 \times 10^{-17}$ \\
\hline & 20 & 0.30 & $2 \times 10^{-17}$ \\
\hline \multirow[t]{2}{*}{ GA2 } & 0 & 0.33 & $4 \times 10^{-17}$ \\
\hline & 20 & 0.28 & $6 \times 10^{-18}$ \\
\hline \multirow[t]{2}{*}{ GA3 } & 0 & 0.49 & $5 \times 10^{-14}$ \\
\hline & 20 & 0.4 & $2 \times 10^{-15}$ \\
\hline \multirow[t]{2}{*}{ GAE1 } & 0 & 0.24 & $2 \times 10^{-18}$ \\
\hline & 60 & 0.29 & $1 \times 10^{-17}$ \\
\hline \multirow[t]{2}{*}{ GAE2 } & 0 & 0.25 & $2 \times 10^{-18}$ \\
\hline & 60 & 0.30 & $2 \times 10^{-17}$ \\
\hline \multirow[t]{2}{*}{ GAE3 } & 0 & 0.43 & $8 \times 10^{-17}$ \\
\hline & 60 & 0.46 & $7 \times 10^{-16}$ \\
\hline \multirow[t]{3}{*}{ GAB1 } & 0 & 0.39 & $1 \times 10^{-17}$ \\
\hline & 20 & 0.39 & $4 \times 10^{-17}$ \\
\hline & 40 & 0.43 & $1 \times 10^{-16}$ \\
\hline \multirow[t]{3}{*}{ GAB2 } & 0 & 0.40 & $1 \times 10^{-16}$ \\
\hline & 20 & 0.41 & $1 \times 10^{-16}$ \\
\hline & 40 & 0.45 & $4 \times 10^{-16}$ \\
\hline
\end{tabular}

* $T=300 \mathrm{~K}$ for samples GA1-GA3, GAE1-GAE3, and $T=340 \mathrm{~K}$ for $\mathrm{GAB} 1$ and $\mathrm{GAB} 2$

$0.43 \mathrm{eV}$ and having a small trapping cross section $\left(10^{-16} \div 10^{-18} \mathrm{~cm}^{2}\right)$ are known in literature (for example, see [11]), but the levels exact atomic configuration is not investigated; they are assumed to relate to the point defects containing an impurity atom. The level $\left(E_{C}-E_{t}\right)=$ $=(0.40 \div 0.42) \mathrm{eV}$ and $\sigma_{n}=\left(10^{-15} \div 10^{-16}\right) \mathrm{cm}^{2}$ is usually assigned to the configuration $V_{\mathrm{Ga}}-V_{\mathrm{As}}$ (EL5 center) $[12,13]$.

On our opinion, the changes of the deep level parameters are related to the structural-impurity alteration of a semiconductor near-surface region, caused by microwave treatment. Such alteration is earlier found out in [5-7]. Namely, according to [5,7], defects are gettered in a material surface layer after this MW irradiation. The charged defects concentration increase must result in the electric field strength increase in this region. Simultaneously, the capture cross section of the levels $E_{C}-0.33 \mathrm{eV}$, $E_{C}-0.31 \mathrm{eV}$ and $\sigma_{n}=\left(10^{-17} \div 10^{-18}\right) \mathrm{cm}^{2}$ is known [11] to decrease when the electric field increase. The cross section decrease is observed for the samples GA1-3. If the free carrier concentration is higher, than the charged defect screening is more effective and, consequently, the change of GA1 $\left(n=10^{18} \mathrm{~cm}^{-3}\right)$ is less significant than that of GA2 $\left(n=10^{16} \mathrm{~cm}^{-3}\right)$. In addition, the samples with high resistance have the larger "skin-depth" of the microwave penetration [14]. The microwave induced increase of the surface curvature radius and the internal mechanical strain relaxation of $n-n^{+}$-GaAs structure nearsurface layers were observed in [5]. It takes place due to the separate dislocation origin and their diffusion along sliding planes toward structure depth. Thus, the changing of value of the both electric and mechanical field intensity take place in the near-surface region, and it, on our opinion, results in the deep level alteration and parameter changing. And, if the changes take place due to the linear and point defects spatial moving, then the microwave influence must depend on crystal orientation (see GA1 and GA3, for example).

It is considered in $[8,9]$ that the trap TAV appearance in the epitaxial structures relates to centers at the internal epilayer-substrate boundary (internal surface). The monocrystal TAV is caused by the defects at the external surface. On our point of view, it is this spatial location difference that is the reason of the MW induced changes character difference (point defects move from sample depth toward external surface, dislocation to opposite side) as well as dose the dependence difference for the epitaxial and monocrystalline wafers.

\section{Conclusions}

The microwave irradiation influence on the electron capture cross section and the energy level of centers in the forbidden gap of $n$-GaAs monocrystal and $n$-GaAs-based epitaxial structures has been investigated for the first time. It was found that the deep level parameters are modified, and the character of changes is different for monocrystals and epitaxial structures. The observed changes is likely to be due to gettering the defects in the material surface layer and internal stress relaxation induced by the microwave treatment applied.

\section{Acknowledgement}

The author would like to thank Prof. R.V. Konakova for helpful discussions.

\section{References}

1. F.P. Korshunov, Yu.V. Bogatirev, V.A. Vavilov, The radiation influence on integrated circuits, Nauka, Minsk (1986) (in Russian).

2. V.V. Kozlovskii, V.A. Kozlov, V.N. Lomasov, Modification of Semiconductors with Proton Beams. A Review // Semiconductors, 34 (2), p. 123-144 (2000).

3. B.N. Zaveryukhin, N.N. Zaveryukhina, R.A. Muminov, O.M. Tursunkulov, Alternating-Strain-Induced Drift of Nonequilibrium Charge Carriers in GaAs Photodetectors // Technical Physics Letters, 28 (3), p. 207-210 (2002). 


\section{O.Ya. Olikh: Acoustoelectric transient spectroscopy of microwave ...}

4. O.Ya. Olikh, I.V. Ostrovskii Ultrasound-Stimulated Increase in the Electron Diffusion Length in p-Si Crystals // Physics of the Solid State, 44(7), p.1249-1253 (2002).

5. N.S. Boltovets, A.B. Kamalov, E.Yu. Kolyadina, R.V. Konakova, P.M. Lytvyn, O.S. Lytvyn, L.A. Matveeva, V.V. Milenin, O.E. Rengelovych, Microwave-Stimulated Relaxation of Internal Strain in GaAs-Based Device Heterostructures // Technical Physics Letters, 28 (2), p. 154-156 (2002).

6. Yu.Yu. Bacherikov, R.V. Konakova, A.N. Kocherov, P.M. Lytvyn, O.S. Lytvyn, O.B. Ohrimenko, A.M. Svetlichnyi, Effect of Microwave Annealing on Silicon Dioxide/Silicon Carbide Structures // Technical Physics, 48(5), p. 598-601 (2003).

7. A.A. Belyaev, A.E. Belyaev, I.B. Ermolovich, S.M. Komirenko, R.V. Konakova, V.G. Lyapin, V.V. Milenin, E.A. Solov'ev, M.V. Shevelev, Influence of microwave treatment on the electrophysical characteristics of technically important semiconductors and surface-barrier structures // Technical Physics, 43(12), p. 1445-1449 (1998).

8. I.V. Ostrovskii, S.V. Saiko, The GaAs surface state spectroscopy by acoustoelectric effect // Fizika Tverdogo Tela, 35(4), p. 1043-1050 (1993).
9. I.V. Ostrovskii, O.Ya. Olikh, Characterization of interface deep levels in as vapor grown epi-GaAs // Solid State Communication, 107(7), p. 341-343 (1998).

10. A.V. Rjanov, The energy spectra of surface state and the kinetics of impulse field effect // Fizika $i$ Tekhnika Poluprovodnikov, 6(8), p. 1495-1501 (1972).

11. F.S. Shishiyanu, Diffusion and degradation into semiconductor materials and devices, Shtiintsa, Kishinev (1978) (in Russian).

12. Yu. Vaitkus, Yu. Storasta, A. Pintsevichus, M. Pjatrauskas, V. Kazjukauskas, The semiisolate GaAs deep level parameters definition by fotoconductivity relaxation after laser excitation // Litovskii fizicheskii sbornic, 28(6), p. 744-751 (1988).

13. V.Ya. Samoilov, N.A. Yakusheva, V.Ya. Prints, The isovalent $\mathrm{Sb}$ impurity influence on arising of electric activity centers in liquid grown epi-n-GaAs // Fizika $i$ Tekhnika Poluprovodnikov, 28(9), p. 1617-1626 (1994).

14. H. Zohm, E. Kasper, P. Mehringer, G.A. Muller, Thermal processing of silicon wafers with microwave co-heating // Microelectronic Engineering, 54, p. 247-253 (2000). 